\title{
Interference Limited MIMO Measurements
}

\author{
S. Jaeckel, L. Thiele and V. Jungnickel \\ Fraunhofer Inst. for Telecommunications, \\ Heinrich Hertz Institute, 10587 Berlin, Germany \\ eMail: \{ stephan.jaeckel ; lars.thiele ; volker.jungnickel \}@hhi.fraunhofer.de
}

\begin{abstract}
We evaluate the interference limited spectral efficiency of a small cellular network using measurements with 3 sites at an ISD of $750 \mathrm{~m}$. Each 3-sector site is equipped with cross-polarized panel antennas. We provide an efficient method to estimate the MIMO capacity from the measured data. We then compare the capacities of SISO and $2 \times 2$ MIMO with and without interference. At a fixed SNR of $10 \mathrm{~dB}$, our cross-polarized interference free $2 \times 2$ setup achieves $5.7 \mathrm{bps} / \mathrm{Hz}$ which compares well to findings from Manhattan. For the best serving BS, the median $2 \times 2$ capacity including the received power and a reuse factor of 9 is $1.7 \mathrm{bps} / \mathrm{Hz}$. This value increases to $3 \mathrm{bps} / \mathrm{Hz}$ when using frequency reuse 3 and $4.7 \mathrm{bps} / \mathrm{Hz}$ with full reuse.
\end{abstract}

\section{INTRODUCTION}

Recently, there are many discussions on how the efficiency of cellular networks can be increased in order to fulfill the rising demands of higher transmission rates. Current technologies reuse the scare resources (e.g. the wireless spectrum) in each cell which leads to a significant amount of intercell interference. At the same time, several approaches for handling this interference are discussed [1]. One open question is: What can be gained in a realistic deployment scenario using real base stations (BSs) and measured channels?

It is a common approach to evaluate different transmission and reception schemes by utilizing system level simulations (e.g. to characterize the throughput of a multiuser multipleinput multiple-output (MIMO) system [2]). The calibration

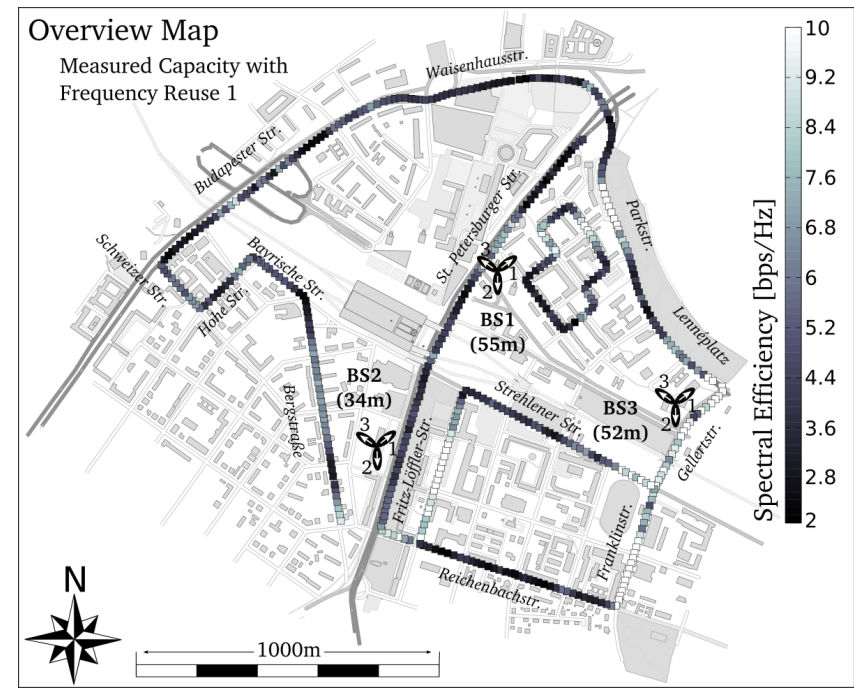

Figure 1. Overview map of the measurement area showing the positions and heights of the BSs, the sector orientations as black leaves and the $2 \times 2$ capacities for the best server with interference from all other sectors. of those simulation environments often relies on channel measurements to estimate the model parameters. However, extensions to the multicell case are often with limited experimental validation. A few measurement results addressing the shadow fading and the angular spread are provided by Jalden et. al. [3] while multicell delay spreads, fading correlations and the cell geometry are evaluated in our earlier paper [4].

One parameter, the spectral efficiency (also called capacity), characterizes the achievable throughput of a channel realization. To benchmark a certain transmission scheme (e.g. Interference Aware Scheduling [5]), knowledge about the channel capacity is of particular importance. However, evaluating the spectral efficiency from measured data is difficult because of the noise. To increase the signal to noise ratio (SNR) one can parameterize the measurement setup to e.g. perform narrowband soundings at a high transmission power [6] or to average several successive snapshots of a wideband sounding [7]. Both methods have their disadvantage because they either limit the frequency- or the doppler resolution. Another approach is to preprocess the data. This can be done by techniques such as ESPRIT or Rimax [8]. While both methods have a high computational complexity, our approach utilizes a much simpler method [9] which is based on adaptive thresholding and weighted averaging over the measurement antennas. The key components if this method are sketched in the appendix.

The preprocessing enables us to evaluate the interference limited spectral efficiency of a virtual 2x2 MIMO setup in a network consisting of $9 \mathrm{BSs}$. The results will help to understand the scaling of the capacity when switching from a cell based single-input single-output (SISO) network with interference avoidance due to a frequency reuse scheme to a MIMO setup with full reuse in each cell. The preprocessing will also allow a direct comparison with linear transmission schemes in simulations (by e.g. replacing the simulated with the processed channels) or the test of prototype hardware using a channel emulator. The set of possible scenarios is, nevertheless, limited to the measurement setup. In the remainder of this paper we describes the experimental setup and the preprocessing method. The results then focus on the capacity of the $2 \times 2$ MIMO link including the interference from neighboring sectors.

\section{EXPERIMENTAL SETUP}

The measurement area is situated in the center of the city of Dresden. Fig. 1 shows the measurement track, the locations of the three BSs with an inter-site distance (ISD) of $750 \mathrm{~m}$ and the 


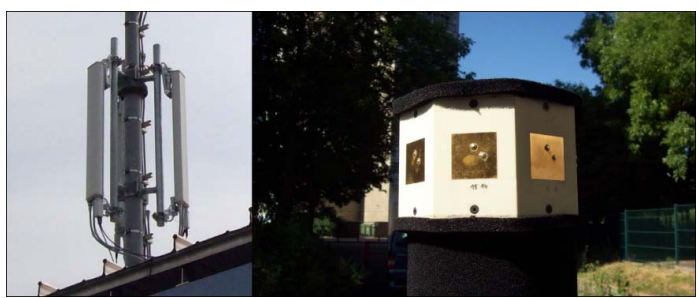

Figure 2. Transmit antenna (left) and receive antenna (right)

sector orientations. All BSs operate at $0^{\circ}$ downtilt during our measurement. A high precision odometer and a GPS receiver record the position of each snapshot. We cluster the snapshots along the $8.8 \mathrm{~km}$ measurement track in 2240 points with a diameter of $33 \lambda$ to increase the precision of the results.

A HyEff channel sounder [10] records the radio channel with a bandwidth of $21.25 \mathrm{MHz}$ at around $2.53 \mathrm{GHz}$ by applying a multi-tone test signal with a duration of $12.8 \mu \mathrm{s}$. We thus estimate the frequency response (FR) of the channel on 273 equidistantly spaced carriers. The single measurement chain of the sounder is consecutively switched to all transmit (Tx) and receive $(\mathrm{Rx})$ antenna elements with the radio channel in between. Snapshot rates and recording times fulfill the channel sampling theorem. At the BS, we use pre-commercial $+18 \mathrm{dBi}$ antennas (Kathrein 80010541) with two cross-polarized ports $\pm 45^{\circ}$ (see Fig. 2). All sector antennas at one site are fed like a single cross-polarized array with six input ports in the same switching sequence. At the receiver, we use a polarized uniform circular array (PUCA) with 8 patch elements [10]. Measurements with the same sounder but at other frequencies and with other antennas are also reported in $[4,7,8,10]$. Further details for this measurement campaign and additional results can be found in our earlier paper [4].

\section{DATA PROCESSING}

The data processing contains 3 steps: The antenna pattern approximation, denoising and the calculation of the capacity. In the first step, we measure the beam patterns of the 16-port receive antenna and calculate two sets of weights, one for the vertical and one for the horizontal polarization. Those weights enable us to combine the measured channel coefficients to

$$
\hat{\mathbf{H}}=\sum_{r=1}^{16}\left(\begin{array}{l}
\mathbf{w}_{r}^{(V)} \mathbf{h}_{r} \\
\mathbf{w}_{r}^{(H)} \mathbf{h}_{r}
\end{array}\right)
$$

The virtual 2-port antenna separates both polarizations with more than $20 \mathrm{~dB}$ decoupling and the shape of its pattern is similar to the pattern of a dipole antenna (see Fig. 3). The effective channel $\hat{\mathbf{H}}$ has two rows representing both polarization directions. A detailed description of the antenna pattern shaping can be fount in appendix A.

In the second step, we denoise the data by first extracting the taps with an adaptive thresholding approach and then we reassemble a noise reduced version of the channel. Donoho and Johnstone [11,12] discovered that an ideal reconstruction of the channel is achieved when there are less taps than carriers in the data (sparse channel) and when we use only taps above a certain threshold for the reconstruction. A detailed test with a simulated Keyhole channel based on Rayleigh fading shows that the estimation and reconstruction gains approx. $10 \mathrm{~dB}$ of SNR in our scenario. The tap estimation and denoising procedure is described in detail in appendix B.

After the preprocessing is done, we calculate the spectral efficiency $C$ measured in units of $\mathrm{bps} / \mathrm{Hz}$ according to the quasistatic block flat-fading MIMO model [13] with

$$
\mathbf{y}_{n}=\mathbf{H}_{n, i} \mathbf{x}_{n, i}+\sum_{\forall k \neq i} \mathbf{H}_{n, k} \mathbf{x}_{n, k}+\mathbf{v}
$$

where $\mathbf{y}_{n}$ is the received signal on subcarrier $n . \mathbf{x}$ is the transmit data vector, $\mathbf{H}_{n, i}$ is the channel matrix for the serving BS $i$ and $\mathbf{v} \sim \mathcal{C N}\left(0, \sigma^{2} \mathbf{I}\right)$ is additive white Gaussian noise (AWGN). Without channel knowledge at the transmitter, the power is equally spread over the $n_{t}$ transmit antennas. The capacity notes

$$
\begin{aligned}
C & =\frac{1}{N} \sum_{n=1}^{N} \log _{2} \operatorname{det}\left(\mathbf{I}+\frac{1}{n_{t} \cdot \eta} \mathbf{Z}_{n}^{-1} \mathbf{H}_{n, i} \mathbf{H}_{n, i}^{H}\right) \\
\eta & =\frac{1}{n_{r} n_{t} N} \sum_{r=1}^{n_{r}} \sum_{t=1}^{n_{t}} \sum_{n=1}^{N}\left|\mathbf{H}_{r, t, n, i}\right|^{2} \\
\mathbf{Z}_{n} & =\frac{1}{\eta}\left(\frac{P_{N}}{n_{r}} \cdot \mathbf{I}+\frac{1}{n_{r} n_{t}} \sum_{\forall k \neq i} \mathbf{H}_{n, k} \mathbf{H}_{n, k}^{H}\right)
\end{aligned}
$$

where $\eta$ is the average path gain, $\mathbf{Z}$ is the interference covariance matrix and $P_{n}$ is the average noise power.

$$
\begin{gathered}
\text { Effective Antenna Patterns } \\
\text { Tx Vertical - Rx Vertical }
\end{gathered}
$$

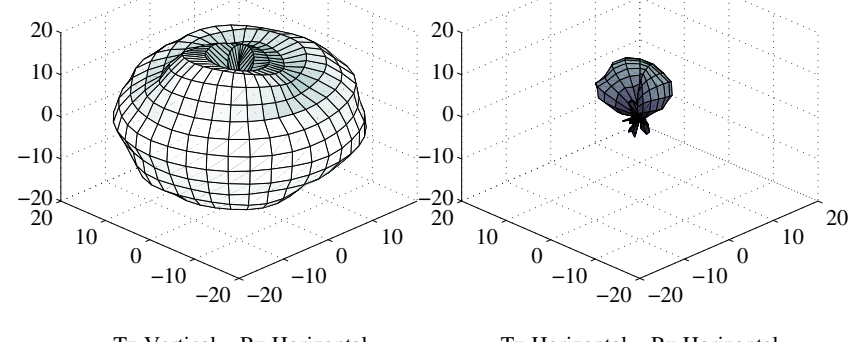

Tx Vertical - Rx Horizontal

Tx Horizontal - Rx Horizontal

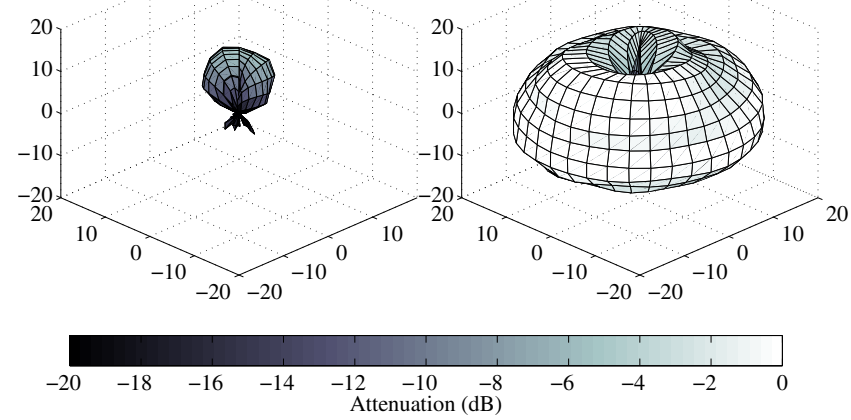

Figure 3. Effective Antenna Patterns. Left: Tx at vertical polarization; Right: Tx at horizontal polarization. 


\section{RESUlts}

\section{A. Capacity of the isolated link}

The 2x2 MIMO capacity is calculated for a fixed SNR of $10 \mathrm{~dB}$. Thus, the results depend only on the structure of the channel matrix and the received power has no influence. All 9575 locations with a raw SNR of above $5 \mathrm{~dB}$ are included in this study and we thus make sure that the potential capacity increase from noise is less than 10\%. Results are depicted in Fig. 4. The median capacity is $5.7 \mathrm{bps} / \mathrm{Hz}$. This result is very close to findings from Chizhik et al. [6] where capacities of $5.5 \mathrm{bps} / \mathrm{Hz}$ are estimated from a narrowband measurement. The capacity distribution (see Fig. 4) indicates that the measured channel matrix is better conditioned than the $2 \times 2$ Rayleigh i.i.d. channel. This can be attributed to the use of cross-polarized antennas. All areas having high capacities above $6.5 \mathrm{bps} / \mathrm{Hz}$ are served by line of sight (LOS). LOS coverage thus provides the highest multiplexing gains for polarized arrays.

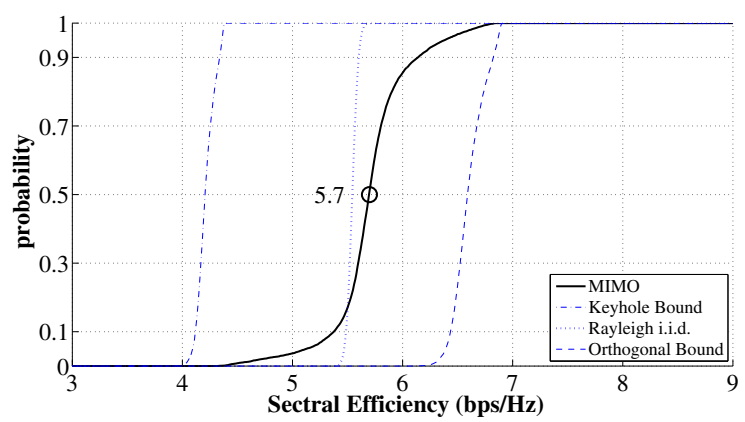

Figure 4. CDF of the spectral efficiency at a fixed SNR of $10 \mathrm{~dB}$.

The maximum throughput is achieved when including both, the effect of the structure of the channel matrix and the received power. For the best server, i.e. the strongest received $\mathrm{BS}$ at the terminal positions, the median $2 \times 2$ capacity is $15 \mathrm{bps} / \mathrm{Hz}$ and the median SNR is $28.4 \mathrm{~dB}$. Best server capacities are about twice as high as the corresponding SISO capacities (median $7.6 \mathrm{bps} / \mathrm{Hz}, 25.3 \mathrm{~dB}$ SNR). The difference in received power can be attributed to the second $\mathrm{Rx}$ antenna for MIMO. Since the Tx array is linearly polarized with $\pm 45^{\circ}$ and the receiver is $\mathrm{V}-\mathrm{H}$, any antenna pair for SISO will have an expected $3 \mathrm{~dB}$ power loss. The measured median difference is $3.1 \mathrm{~dB}$ (see Fig. 6, left). In order to make the results more

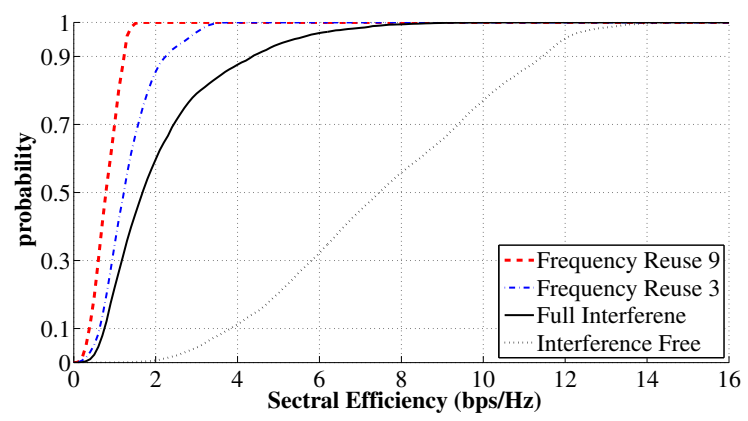

Figure 5. Interference limited SISO capacities including the reuse factor
Table I

MEDIAN INTERFERENCE LIMITED CAPACITIES

\begin{tabular}{|l|r|r|r|}
\hline Transmission scheme & \multicolumn{1}{|c|}{$C$} & $C_{\text {eff }}$ & Gain \\
\hline SISO Interference free & 7.63 & 0.85 & 0.88 \\
Rx comb. Interference free & 8.51 & 0.95 & 1.00 \\
MIMO 2x2 Interference free & 14.98 & 1.66 & 1.72 \\
\hline SISO Diff. res. per sector (reuse 3) & 3.80 & 1.27 & 0.82 \\
Rx comb. Diff. res. per sector (reuse 3) & 4.70 & 1.57 & 1.00 \\
MIMO 2x2 Diff. res. per sector (reuse 3) & 8.99 & 3.00 & 1.87 \\
\hline SISO Full interference (reuse 1) & 1.75 & 1.75 & 0.74 \\
Rx comb. Full interference (reuse 1) & 2.40 & 2.40 & 1.00 \\
MIMO 2x2 Full interference (reuse 1) & 4.69 & 4.69 & 1.94 \\
\hline
\end{tabular}
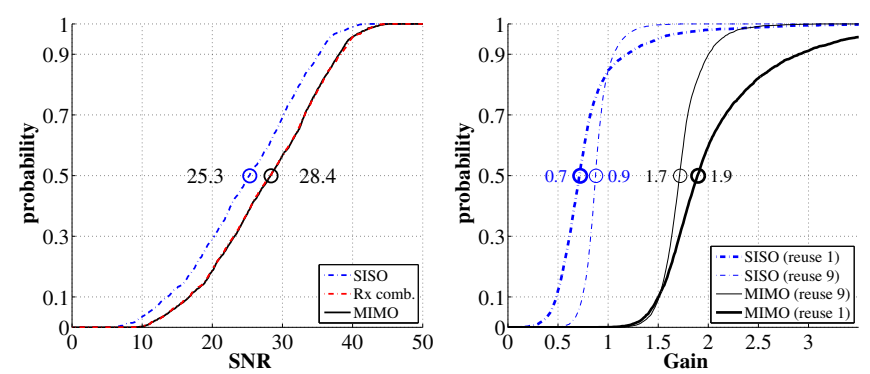

Figure 6. Left: SNR distribution for SISO (blue, dashed) and MIMO (black, solid). Right: Relative capacity gains for frequency reuse 1 (thick lines) and 9 (thin lines). The reference value is the SISO capacity with the signals of both polarizations added.

comparable, we added the signals from both ports of the virtual antenna before calculating the SISO capacity (referred to as Rx combining in Fig. 6 and Tab. I). This removes the polarization dependency and the power gap.

\section{B. Capacity with Interference}

For the interference limited case, we use the strongest received BS for our serving link. Channels from other BSs cause frequency selective interference. In a first scenario, we apply frequency reuse 3 in a way that all sectors facing the same direction (i.e. all sectors facing south) use the same resources. Here, we achieve a median $2 \times 2$ capacity of $9 \mathrm{bps} / \mathrm{Hz}$, while SISO achieves only $3.8 \mathrm{bps} / \mathrm{Hz}$. In the second scenario, we reuse the same resources at each BS. In this case, the median $2 \times 2$ spectral efficiency is $4.7 \mathrm{bps} / \mathrm{Hz}$ while SISO only achieves $1.75 \mathrm{bps} / \mathrm{Hz}$ (see Fig. 7, black solid line). This does not compare to the interference free link since we have to apply the reuse factor. If we apply reuse 9 in our network (and thus achieve only $\frac{14.98}{9} \approx 1.66 \mathrm{bps} / \mathrm{Hz}$ for MIMO), then we can achieve a roughly three times higher capacity by applying frequency reuse 1 (see column $C_{\text {eff }}$ in Tab. I).

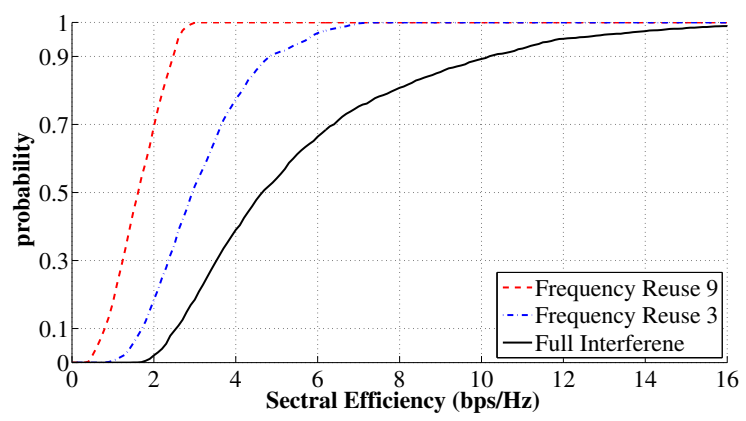

Figure 7. Interference limited $2 \times 2$ capacities including the reuse factor 
An interesting observation is that with increasing interference, the capacity gap of SISO and MIMO also increases. This can be seen in the last column of Tab. I where the relative gains compared the Rx combining case are calculated. In other words - when switching from frequency reuse 9 to reuse 1 , the effective capacity for SISO increases roughly by a factor of 2 while for MIMO, the factor is 3 . The distribution of the relative gains is depicted in Fig. 6, right.

\section{Summary AND Discussion}

We show that data processing can provide more detailed insights into the performance of cellular MIMO systems. We can remove a significant part of the noise and thus evaluate the broadband MIMO channel capacity for a mobile network. Our results confirm prior findings from narrowband measurements in an isolated cell in Manhattan and we can provide reliable estimates also when the measurement SNR is low.

Compared to frequency reuse 9, 2x2 MIMO capacities are three times higher when all base stations operate on the same resources. Our results also show an advantage of MIMO over conventional SISO systems. First, cross-polarized 2x2 MIMO does not lose power when the polarization directions of transmitter and receiver are not aligned at each other. Second, intelligent signal processing in space and frequency domain can handle interference much more efficient when using multiple antennas. For SISO, interference can be seen as a simple additional power distorting the serving signal. For MIMO, nevertheless, the interference covariance is normally non-white and has different values for each link and frequency bin. The combination of those effects - polarization and interference - leads to a 2.5 times higher capacity of MIMO compared to SISO. This can not be observed in the interference free link where the factor is 1.97. But note that in our setup the polarization was kept constant during all the drive tests. Also note that our findings are only valid for a limited network where there is no outer interference. Future work will focus on both, the comparison of our findings with system level simulations and the gains of $\mathrm{BS}$ cooperation in a statistical scenario.

\section{APPENDIX}

\section{A. Antenna pattern shaping}

The beam patterns of the receive array are measured in an anechoic chamber with one degree precision in azimuth and

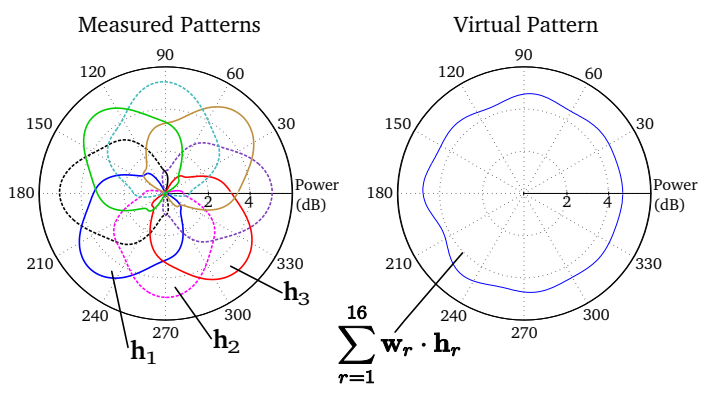

Figure 8. Measured and virtual $\mathrm{V}-\mathrm{V}$ pattern at $0^{\circ}$ elevation elevation. Two separate measurements are done for the horizontal and vertical polarization. We use a linear combination of each of the 16 elements of the receive array to emulate omnidirectional coverage. This gives more representative results and provides an additional array gain for the data evaluation. The linear weights are calculated separately for each polarization. We first assemble a matrix $\mathbf{P}$ containing all measured patterns of the same polarization at 0 degree elevation. E.g. we get $\mathbf{P}^{(\mathrm{V})}$ with 8 rows, one for each patch element, and 360 columns representing the measured beam patterns where the Tx and the $\mathrm{Rx}$ are aligned at vertical polarization. Likewise, we get $\mathbf{P}^{(\mathrm{H})}$ by using the patterns with horizontal alignment. In the second step, we calculate the Moore-Penrose pseudoinverse of $\mathbf{P}^{(\mathrm{V})}$ and $\mathbf{P}^{(\mathrm{H})}$

$$
\mathbf{P}^{-1}=\mathbf{P}^{H} \cdot\left(\mathbf{P} \mathbf{P}^{H}\right)^{-1}
$$

Now, we provide a new beam pattern with our desired shape. We want to emulate an omnidirectional 1x360 pattern given as a vector of ones. Note here, that theoretically any pattern can be approximated in this way. The approximation accuracy solely depends on the number of elements at the measurement antenna. The weights are now calculated by multiplying our desired pattern with $\mathbf{P}^{-1}$

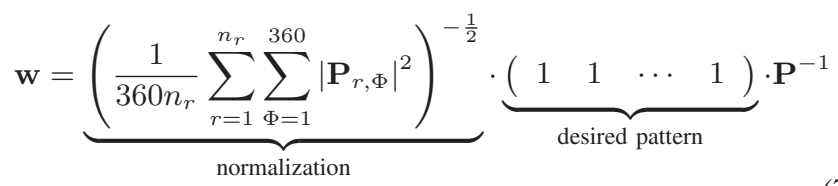

This calculation is done separately for both polarizations resulting in two weight vectors $\mathbf{w}^{(\mathrm{V})}$ and $\mathbf{w}^{(\mathrm{H})}$ for the vertical and horizontal alignment, respectively. In a last step, we apply the two weight vectors to the measured pattern at all azimuth and elevation angles to calculate the effective beam pattern of the virtual antenna. The result is depicted in Fig. 3.

\section{B. Denoising by tap estimation}

Both, the measured and the effective channel coefficients can be seen as

$$
\mathbf{y}_{n}=\mathbf{h}_{n}+\mathbf{v}_{n}, \quad n \in\{1,2, \ldots N\}
$$

where $\mathbf{y}$ is the observation of the broadband radio channel $\mathbf{h}$ in frequency domain at $N$ fixed pilot positions $\nu_{n}$ with $\nu_{n} \in \mathbb{R}$ and $0 \leq \nu_{n}<1$. Note that the pilot positions do not need to be equidistant. $\mathbf{v}$ is assumed to be AWGN. We further assume that the FR of the channel can be modelled as a sum of discrete reflections (channel filter taps) of the transmitted waveform at obstacles in the physical environment [14]. We want to estimate the taps from the observation $\mathbf{y}$ in an iterative fashion in order to find a representation $\hat{\mathbf{h}}$ of the channel where most of the noise has been removed. In each iteration step, we estimate the normalized delay $m_{l}=\tau_{l} \cdot B$ of the $l^{\text {th }}$ tap by

$$
m_{1}=\arg \max _{m_{l}}\left|\sum_{n=1}^{N} \mathbf{g}_{n} \cdot e^{-2 \pi j \cdot m_{l} \cdot \nu_{n}}\right|
$$




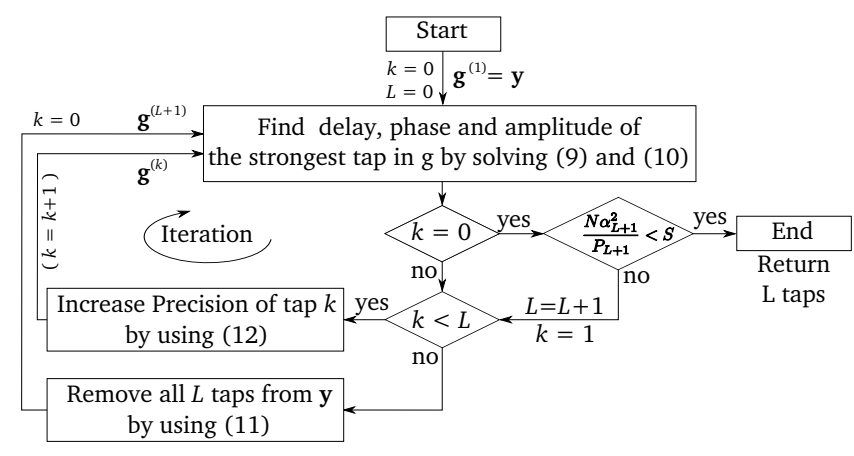

Figure 9. Flow chart of the iterative tap detection with adaptive thresholding. The inner iteration loop can be repeated more then once for each new detected tap - resulting in further precision improvements.

For the first step we use $\mathbf{g}_{n}=\mathbf{y}_{n}$ from (8). $m_{l} \in \mathbb{R}$ can have values $0 \leq m_{l}<\tau_{m} \cdot B$ with $\tau_{m}$ as the maximum delay in the measurement. For the amplitude $\alpha_{l}$ and phase $\phi_{l}$ follows

$$
\alpha_{l} \cdot e^{j \phi_{l}}=\frac{1}{N} \cdot \sum_{n=1}^{N} \mathbf{g}_{n} \cdot e^{-2 \pi j \cdot m_{l} \cdot \nu_{n}}
$$

In further iteration steps we can set $\mathbf{g}_{n}$ in two ways:

1) We want to estimate a new tap and we know $L$ taps already. Therefore we remove all previously detected taps from the observation $\mathbf{y}$.

$$
\mathbf{g}_{n}^{(L+1)}=\mathbf{y}_{n}-\sum_{l=1}^{L} \alpha_{l} \cdot e^{j \phi_{l}} \cdot e^{-2 \pi j \cdot m_{l} \cdot \nu_{n}}
$$

2) The values $\alpha, \phi$ and $\tau$ of each tap are distorted by interference from the following (undetected) taps and also by approximation errors of already estimated ones. After we estimated a new tap, we can increase the precision of a previously detected tap $k$ by setting $\mathbf{g}$ to

$$
\mathbf{g}_{n}^{(\mathrm{k})}=\mathbf{y}_{n}-\sum_{\forall l \neq k}^{L} \alpha_{l} \cdot e^{j \phi_{l}} \cdot e^{-2 \pi j \cdot m_{l} \cdot \nu_{n}}
$$

and repeating the calculation for this tap. Candes and Tao [15] state that for a sparse $\mathbf{h}$, which is satisfied when there are less taps $L$ then observations $N$, we can find a $\hat{\mathbf{h}}$ such that the mean square error (MSE) is within a factor of $\log N$ of the ideal MSE

$$
\|\hat{\mathbf{h}}-\mathbf{y}\|^{2}=O(\log N) \cdot \mathrm{E}\|\mathbf{y}-\mathbf{h}\|^{2}
$$

Donoho and Johnstone $[11,12]$ further state that thresholding achieves the ideal MSE with a threshold level at $\sqrt{2 \cdot \log N} \cdot \sigma$. In general, the exact number of taps is unknown. We can gain knowledge about the noise power $P_{N}$, and thus the threshold from the link budget in the measurement setup. Nevertheless, if the exact value $P_{N}$ is also unknown and we can certify that $L \ll N$, then we can set the threshold in an adaptive fashion. In this case, when a new tap is found, we calculate the power that is left after removing all detected taps according to

$$
P_{L+1}=\frac{1}{N} \sum_{n=1}^{N}\left(\mathbf{y}_{n}-\sum_{l=1}^{L+1} \alpha_{l} \cdot e^{j \phi_{l}} \cdot e^{-2 \pi j \cdot m_{l} \cdot \nu_{n}}\right)^{2}
$$

When there are $L$ taps above and the $(L+1)^{\text {st }}$ tap falls below threshold and it is provided that $|\mathbf{v}|^{2} \sim \chi_{2}^{2}$, then we can set $\sigma=2 \cdot P_{L+1}$ and stop the iterative estimation when

$$
\alpha_{L+1}^{2}<\underbrace{2 \cdot \sqrt{2 \cdot \log N}}_{=S} \cdot \frac{P_{L+1}}{N}
$$

$S$ is the threshold level and the additional factor $1 / N$ comes from the time domain representation (10) while (14) is in frequency domain. Note that when (15) is fulfilled, the $(L+1)^{\text {st }}$ tap is not part of $\hat{\mathbf{h}}$. A flow chart of the iterative estimation algorithm is depicted in Fig. 9.

\section{ACKNOWLEDGMENTS}

The authors wish to thank the German Ministry of Education and Research (BMBF) for financial support in the national collaborative project EASY-C under contract number 01BU0631. Furthermore we thank our partners Actix, Alcatel-Lucent, Deutsche Telekom, Ericsson, HHI, Kathrein, Qualcomm, TU Dresden and Vodafone for financial support of the campaign in Dresden. Many thanks to C. Schneider, G. Sommerkorn (all from TU Ilmenau) and S. Warzügel (MEDAV) for assistance during the measurements and $\mathrm{M}$. Schellmann from HHI for the fruitful discussions.

\section{REFERENCES}

[1] J. Andrews, W. Choi, and R. Heath Jr., "Overcoming interference in spatial multiplexing MIMO cellular networks," IEEE Wireless Commun., vol. 14, no. 6, pp. 95-104, 2007.

[2] L. Thiele, M. Schellmann, W. Zirwas, and V. Jungnickel, "Capacity scaling of multiuser MIMO with limited feedback in a multicell environment," Proc. ACSSC '07, 2007.

[3] N. Jaldén, P. Zetterberg, B. Ottersten, and L. Garcia, "Inter-and intrasite correlations of large-scale parameters from macrocellular measurements at $1800 \mathrm{MHz}, "$ EURASIP JWCN, vol. 2007, no. 3, 2007.

[4] S. Jaeckel, L. Thiele, A. Brylka, L. Jiang, and V. Jungnickel, "Intercell interference measured in urban areas," Proc. IEEE ICC '09, 2009.

[5] V. Jungnickel, M. Schellmann, L. Thiele, T. Wirth, T. Haustein, O. Koch, E. Zirwas, and E. Schulz, "Interference aware scheduling in the multiuser MIMO-OFDM downlink," IEEE Commun. Mag., vol. 47, pp. 56-66, June 2009.

[6] D. Chizhik, J. Ling, P. Wolniansky, R. Valenzuela, N. Costa, and K. Huber, "Multiple-input-multiple-output measurements and modeling in Manhattan," IEEE J. Sel. Areas Commun., vol. 21, pp. 321-331, 2003.

[7] V. Jungnickel, S. Jaeckel, L. Thiele, L. Jiang, U. Krüger, A. Brylka, and C. Helmolt, "Capacity measurements in a cooperative multicell MIMO network," IEEE Trans. Veh. Technol., vol. 58, pp. 2392-2405, 2009.

[8] A. Richter, "Estimation of radio channel parameters: Models and algorithms," Ph.D. dissertation, Teschniche Universität Ilmenau, 2005.

[9] S. Jaeckel and V. Jungnickel, "Estimating MIMO capacities from broadband measurements in a cellular network," Proc. EUCAP '10, 2010.

[10] R. Thomä, D. Hampicke, A. Richter, G. Sommerkorn, and U. Trautwein, "MIMO vector channel sounder measurement for smart antenna system evaluation," Europ. Trans. Telecommun., vol. 12, pp. 427-438, 2001.

[11] D. L. Donoho and I. M. Johnstone, "Ideal denoising in an orthonormal basis chosen from a library of bases," Comptes Rendus Acad. Sci., Ser. I, vol. 319, pp. 1317-1322, 1994.

[12] — " "Ideal spatial adaptation by wavelet shrinkage," Biometrika, vol. 81, pp. 425-455, 1994.

[13] E. A. Jorswieck and H. Boche, "Performance analysis of capacity of MIMO systems under multiuser interference based on worst-case noise behavior," EURASIP JWCN, vol. 2, pp. 273-285, 2004.

[14] D. Tse and P. Viswanath, Fundamentals of Wireless Communication. Cambridge University Press, 2005.

[15] E. Candes and T. Tao, "The dantzig selector: Statistical estimation when p is much larger than n," Ann. Statist., vol. 35, pp. 2313-2351, 2007. 